\title{
The uniqueness and well-posedness of vector equilibrium problems with a representation theorem for the solution set
}

\author{
Ding-Tao Peng ${ }^{1 *}$, Jian Yu and Nai-Hua Xiu ${ }^{2}$
}

\author{
"Correspondence: \\ dingtaopeng@126.com \\ ${ }^{1}$ College of Science, Guizhou \\ University, Guiyang, Guizhou \\ 550025, China \\ Full list of author information is \\ available at the end of the article
}

\begin{abstract}
This paper aims to present some uniqueness and well-posedness results for vector equilibrium problems (for short, VEPs). We first construct a complete metric space $M$ consisting of VEPs satisfying some conditions. Using the method of set-valued analysis, we prove that there exists a dense everywhere residual subset $Q$ of $M$ such that each VEP in $Q$ has a unique solution. Moreover, we introduce and obtain the generalized Hadamard well-posedness and generic Hadamard well-posedness of VEPs by considering the perturbations of both vector-valued functions and feasible sets. As an application, we provide a representation theorem for the solution set to each VEP in $M$.
\end{abstract}

MSC: 49K40; 90C31;46B40;47H04

Keywords: vector equilibrium problem; set-valued mapping; dense everywhere residual set; uniqueness; well-posedness

\section{Introduction}

The vector equilibrium problem (for short, VEP) is a natural generalization of the equilibrium problem for the vector-valued function. It is well known that the vector equilibrium problem is a unified model of several fundamental mathematical problems, namely, the vector optimization problem, the vector variational inequality, the vector complementarity problem, the multiobjective game, the vector network equilibrium problem etc. Since the VEP was proposed at about 1997 lots of peoples have made many contributions to this problem and hundreds of papers have been published; see, e.g., the collection [1] and the monograph [2]. However, works on the uniqueness of solutions to VEPs were hardly seen. The only work we can find about the uniqueness of solutions to VEPs is [3], in which Khanh and Tung established sufficient conditions for the local uniqueness of solutions to VEPs by using approximations as generalized derivatives under the assumption that the functions have first and second Fréchet derivatives. The reason why results on uniqueness are so few is due to the fact that except for a few types of mathematical problems, most of the mathematical problems cannot guarantee the uniqueness of the solution. Therefore, to consider the generic uniqueness of the solutions may be more suitable, which will answer the question how many problems there are in a class of problems having a unique solution. The generic uniqueness of solutions to VEPs is our main motivation in this paper.

As we know, several works have been achieved about the generic uniqueness of solutions to some optimization-related problems such as optimization problems $[4,5]$, two-person

\section{空 Springer}

C2014 Peng et al: licensee Springer. This is an Open Access article distributed under the terms of the Creative Commons Attribution License (http://creativecommons.org/licenses/by/2.0), which permits unrestricted use, distribution, and reproduction in any medium, provided the original work is properly cited. 
zero-sum continuous games [6], saddle point problems [7], large crowding games [8]. Recently, some new results were obtained. Yu et al. [9] obtained the generic uniqueness of equilibrium points for a class of equilibrium problems. The results in [9] showed that most of the monotone equilibrium problems (in the sense of Baire category) have a unique equilibrium point and that each monotone equilibrium problem can be arbitrarily approached by a sequence of such equilibrium problems that each of them has a unique equilibrium point. Moreover, Peng et al. [10] provided a unified approach to the generic uniqueness and applied it to several nonlinear problems. However, the study of the generic uniqueness of solutions to VEPs has an essential difficulty: that the values of different vector-valued functions are incomparable. To overcome such a difficulty is one of the main tasks in this paper.

The stability of solutions to nonlinear problems is also an important topic. The notion of well-posedness is just one of the approaches to the stability. There have been several notions of well-posedness about optimization-related problems. We refer to [11-15] for more details. For well-posedness of equilibrium problems or vector equilibrium problems, there are some results. Fang et al. [16] investigated the well-posedness of equilibrium problems; Kimura et al. [17] studied the parametric well-posedness for vector equilibrium problems; Bianchi et al. [18] introduced and studied two types of well-posedness for vector equilibrium problems; Li and Li [19] investigated the Levitin-Polyak well-posedness of vector equilibrium problems with variable domination structures; Salamon [20] analyzed the Hadamard well-posedness of parametric vector equilibrium problems; Peng et al. [21] investigated several types of Levitin-Polyak well-posedness of generalized vector equilibrium problems. Most of these works considered the perturbation of the parameters in the vector-valued functions. Different from these works, we will not only consider the perturbation of objective functions but also consider the perturbation of feasible sets.

This paper aims to present some generic uniqueness and well-posedness results for VEPs. We consider both the perturbation of vector-valued functions and the perturbation of feasible sets. The paper is organized as follows. In Section 2, we recall some definitions and preliminaries. In Section 3, we investigate the uniqueness of solutions to VEPs. We first construct a complete metric space $M$ consisting of VEPs satisfying some conditions. Then we prove that most of the VEPs (in the sense of Baire category) in $M$ have a unique solution. In Section 4, the Hadamard well-posedness of VEPs is introduced and studied. The generalized Hadamard well-posedness and generic Hadamard well-posedness of VEPs are derived. In Section 5, applying the above results we provide an interesting representation theorem for the solution set of each VEP in $M$. Finally, we briefly conclude our results in Section 6.

\section{Preliminaries}

Throughout this section, let $H$ be a Hausdorff topological vector space and $C$ be a nonempty closed, convex and pointed cone in $H$ with int $C \neq \emptyset$, where int $C$ denotes the topological interior of $C$. We note that int $C+C \subset \operatorname{int} C$ (see [22]).

Let $X$ be a nonempty set and $\phi: X \times X \rightarrow H$ be a vector-valued function. The so-called vector equilibrium problem (for short, VEP) [2] is to find $x^{*} \in X$ such that

$$
\phi\left(x^{*}, y\right) \notin-\operatorname{int} C, \quad \forall y \in X .
$$


We call $x^{*}$ a solution of $\operatorname{VEP}(\phi)$. If $H=\mathbb{R}, C=(-\infty, 0]$, the VEP becomes the Ky Fan inequality [23, 24]. Similarly, if $H=\mathbb{R}, C=[0,+\infty)$, the VEP becomes the equilibrium problem [25].

Definition 2.1 (see [2]) Let $X$ be a nonempty subset of a Hausdorff topological vector space $E$ and $f: X \rightarrow H$ be a vector-valued function. $f$ is said to be $C$-upper semicontinuous at $x \in X$ iff for any open neighborhood $V$ of 0 in $H$, there exists an open neighborhood $U$ of $x$ in $X$ such that, for any $x^{\prime} \in U$,

$$
\left.f\left(x^{\prime}\right) \in f(x)+V-C \quad \text { (or equivalently, } f(x) \in f\left(x^{\prime}\right)+V+C\right) ;
$$

$f$ is said to be $C$-upper semi-continuous on $X$ iff $f$ is $C$-upper semi-continuous at each $x \in X$; and $f$ is said to $C$-lower semi-continuous on $X$ iff $-f$ is $C$-upper semi-continuous on $X$.

Definition 2.2 Let $X$ be a nonempty subset of a Hausdorff topological vector space $E$ and $\phi: X \times X \rightarrow H$ be a vector-valued function. $\phi$ is said to be $C$-strictly-quasi-monotone on $X \times X$ iff for any $x, y \in X$ with $x \neq y$,

$$
\phi(x, y) \notin-\operatorname{int} C \quad \Rightarrow \quad \phi(y, x) \in-C .
$$

Example 2.3 Let $E=\mathbb{R}, X=[-1,1] \subset E, H=\mathbb{R}^{2}$ and $C=\mathbb{R}_{+}^{2} \subset H$. Define

$$
\begin{aligned}
& f_{1}(x):= \begin{cases}(0,0)^{\top}, & -1 \leq x<0, \\
(1,1)^{\top}, & 0 \leq x \leq 1 ;\end{cases} \\
& f_{2}(x):=(x, x)^{\top}, \quad-1 \leq x \leq 1 ; \\
& \phi(x, y):=(|x|-|y|,|x|-|y|)^{\top}, \quad-1 \leq x, y \leq 1 .
\end{aligned}
$$

One can easily check that $f_{1}$ is $C$-upper semi-continuous on $X$ but not $C$-lower semicontinuous at $x=0 ; f_{2}$ is both $C$-upper semi-continuous and $C$-lower semi-continuous on $X$; $\phi$ is both $C$-upper semi-continuous and $C$-lower semi-continuous on $X \times X$; $\phi$ is $C$-strictly-quasi-monotone on $X \times X$; and that $x= \pm 1 \in X$ are the only two solutions to $\operatorname{VEP}(\phi)$.

To investigate the uniqueness of solutions to VEPs, we will use the way of set-valued analysis. So let us recall some definitions and lemmas about set-valued mappings; for more details see [26].

Definition 2.4 Let $X, M$ be two topological spaces. Denote by $2^{X}$ the space of all nonempty subsets of $X$. Let $S: M \rightarrow 2^{X}$ be a set-valued mapping. Then (i) $S$ is said to be upper (respectively, lower) semi-continuous at $u \in M$ iff for each open set $G$ in $X$ with $G \supset S(u)$ (respectively, $G \cap S(u) \neq \emptyset$ ), there exists an open neighborhood $O$ of $u$ such that $G \supset S\left(u^{\prime}\right)$ (respectively, $G \cap S\left(u^{\prime}\right) \neq \emptyset$ ) for each $u^{\prime} \in O$; (ii) $S$ is said to be continuous at $u \in M$ iff $S$ is both upper semi-continuous and lower semi-continuous at $u$; (iii) $S$ is said to be a usco mapping iff $S$ is upper semi-continuous on $M$ and $S(u)$ is compact for each $u \in M$; (iv) A subset $Q$ of $M$ is called residual iff it contains the intersection of countably many dense everywhere open subsets of $M$. 
Lemma 2.5 (see $[7,27])$ Let $M$ be a Baire space, $X$ be a metric space and $S: M \rightarrow 2^{X}$ be a usco mapping, then there exists a dense everywhere residual subset $Q$ of $M$ such that $S$ is lower semi-continuous at each $x \in Q$.

Remark 2.6 If there exists a dense everywhere residual subset $Q$ of $M$ such that, for each $u \in Q$, a certain property $P$ depending on $u$ holds, then we say that the property $P$ is generic on $M$. Since $Q$ is a second category set, we may say that the property $P$ holds for most of the points (in the sense of Baire category) in $M$. The research on generic properties (including generic existence, generic uniqueness, generic stability, generic well-posedness and so on) has attracted much attention; see, e.g., [4-7, 9, 22, 24, 28, 29] and the references therein.

Lemma 2.7 (see [28]) Let $A$ and $A_{n}(n=1,2, \ldots)$ all be nonempty compact subsets of a metric space $X$ with $A_{n} \rightarrow A$ in the Hausdorff distance topology, then the following statements hold:

(i) $\bigcup_{n=1}^{+\infty} A_{n} \cup A$ is also nonempty compact subset of $X$;

(ii) If $x_{n} \in A_{n}, x_{n} \rightarrow x$, then $x \in A$.

\section{Uniqueness of solutions to VEPs}

In the rest of this paper, let $X$ be a nonempty and closed subset of a complete metric space $E,(H,\|\cdot\|)$ be a Banach space, and $C$ be a nonempty, closed, convex, and pointed cone in $H$ with int $C \neq \emptyset$. For any $\epsilon>0$, denote by $B(\epsilon):=\{z \in H:\|z\| \leq \epsilon\}$ and $B^{\circ}(\epsilon):=\{z \in H$ : $\|z\|<\epsilon\}$. We emphasize that the open neighborhood $V$ in Definition 2.1 can be replaced by $B^{\circ}(\epsilon)$ in the case that $H$ is a normed space.

Let a space $M$ of VEPs be defined by

$$
M=\left\{\begin{array}{l}
u=(\phi, A): \\
\phi \sup _{(x, y) \in X \times X}\|\phi(x, y)\|<+\infty ; \\
A \text { is a nonempty compact subset of } X ; \\
\text { and } \exists x \in A \text { such that } \phi(x, y) \notin-\operatorname{int} C, \forall y \in A
\end{array}\right\} .
$$

For any $u_{1}=\left(\phi_{1}, A_{1}\right), u_{2}=\left(\phi_{2}, A_{2}\right) \in M$, define

$$
\rho\left(u_{1}, u_{2}\right)=\sup _{(x, y) \in X \times X}\left\|\phi_{1}(x, y)-\phi_{2}(x, y)\right\|+h\left(A_{1}, A_{2}\right),
$$

where $h$ is the Hausdorff distance on $X$.

Lemma $3.1(M, \rho)$ is a complete metric space.

Proof Clearly, $\rho$ is a metric on $M$. We only need to show that $(M, \rho)$ is complete. Let $\left\{u_{n}=\left(\phi_{n}, A_{n}\right)\right\}$ be a Cauchy sequence of $M$, then for any $\epsilon>0$, there exists a positive integer $N(\epsilon)$ such that

$$
\rho\left(u_{m}, u_{n}\right)=\sup _{(x, y) \in X \times X}\left\|\phi_{m}(x, y)-\phi_{n}(x, y)\right\|+h\left(A_{m}, A_{n}\right)<\epsilon, \quad \forall m, n \geq N(\epsilon) .
$$


Hence

$$
\sup _{(x, y) \in X \times X}\left\|\phi_{m}(x, y)-\phi_{n}(x, y)\right\|<\epsilon \quad \text { and } \quad h\left(A_{m}, A_{n}\right)<\epsilon, \quad \forall m, n \geq N(\epsilon) .
$$

Since $H$ is a Banach space, for any $x, y \in X$, there exists $\phi(x, y) \in H$ such that $\lim _{m \rightarrow \infty} \phi_{m}(x$, $y)=\phi(x, y)$ and

$$
\sup _{(x, y) \in X \times X}\left\|\phi_{n}(x, y)-\phi(x, y)\right\| \leq \epsilon, \quad \forall n \geq N(\epsilon) .
$$

Since $X$ is complete, $K(X)$ is also complete, where $K(X)$ denotes the space of all nonempty compact subsets of $X$ and is endowed with the Hausdorff distance $h$ induced by the metric on $X$. Consequently, by $h\left(A_{m}, A_{n}\right)<\epsilon$, there exists $A \in K(X)$ such that $A_{n} \rightarrow A$. Next, we will prove $u:=(\phi, A) \in M$.

(i) Fix $n \geq N(\epsilon)$. Since $\phi_{n}$ is $C$-upper semi-continuous on $X \times X$, there exists a neighborhood $U(x, y) \subset X \times X$ of $(x, y)$ such that

$$
\phi_{n}\left(x^{\prime}, y^{\prime}\right) \in \phi_{n}(x, y)+B^{\circ}(\epsilon)-C, \quad \forall\left(x^{\prime}, y^{\prime}\right) \in U(x, y) .
$$

Thus, by (3.1) and (3.2), for any $\left(x^{\prime}, y^{\prime}\right) \in U(x, y)$, we have

$$
\phi\left(x^{\prime}, y^{\prime}\right) \in \phi_{n}\left(x^{\prime}, y^{\prime}\right)+B(\epsilon) \subset \phi_{n}(x, y)+B^{\circ}(2 \epsilon)-C \subset \phi(x, y)+B^{\circ}(3 \epsilon)-C .
$$

It follows that $\phi$ is $C$-upper semi-continuous on $X \times X$.

(ii) For any $x, y \in X$ with $x \neq y$, suppose $\phi(x, y) \notin-\operatorname{int} C$. Since int $C$ is open and $\lim _{n \rightarrow \infty} \phi_{n}(x, y)=\phi(x, y)$, we have $\phi_{n}(x, y) \notin-\operatorname{int} C$ when $n$ is big enough. It follows from the $C$-strictly-quasi-monotonicity of $\phi_{n}$ that $\phi_{n}(y, x) \in-C$. Since $C$ is closed and $\lim _{n \rightarrow \infty} \phi_{n}(y, x)=\phi(y, x)$, we obtain $\phi(y, x) \in-C$. Therefore, $\phi$ is $C$-strictly-quasimonotone on $X \times X$.

(iii) For each $n \geq N(\epsilon)$, we have

$$
\sup _{(x, y) \in X \times X}\left\|\phi_{n}(x, y)-\phi(x, y)\right\| \leq \epsilon \quad \text { and } \quad \sup _{(x, y) \in X \times X}\left\|\phi_{n}(x, y)\right\|<+\infty .
$$

Hence $\sup _{(x, y) \in X \times X}\|\phi(x, y)\| \leq \sup _{(x, y) \in X \times X}\left\|\phi_{n}(x, y)\right\|+\epsilon<+\infty$.

(iv) Since $u_{n}=\left(\phi_{n}, A_{n}\right) \in M$ for each $n=1,2, \ldots$, there exists $x_{n} \in A_{n}$ such that

$$
\phi_{n}\left(x_{n}, y\right) \notin-\operatorname{int} C, \quad \forall y \in A_{n} .
$$

Since $A$ and $A_{n}(n=1,2, \ldots)$ are all compact and $A_{n} \rightarrow A$, by Lemma $2.7(\mathrm{i}), \bigcup_{n=1}^{+\infty} A_{n} \cup A$ is also compact. Note that $\left\{x_{n}\right\} \subset \bigcup_{n=1}^{+\infty} A_{n} \cup A$. Without loss of generality, suppose $x_{n} \rightarrow x^{*}$. By Lemma 2.7(ii), $x^{*} \in A$. We shall show that $x^{*}$ fulfills

$$
\phi\left(x^{*}, y\right) \notin-\operatorname{int} C, \quad \forall y \in A .
$$

Assume, by contradiction, that there exists $y_{0} \in A$ such that $\phi\left(x^{*}, y_{0}\right) \in-\operatorname{int} C$. Since int $C$ is open, there exists $\epsilon_{0}>0$ such that

$$
\phi\left(x^{*}, y_{0}\right)+B^{\circ}\left(2 \epsilon_{0}\right) \subset-\operatorname{int} C .
$$


For $y_{0} \in A$, by virtue of $A_{n} \rightarrow A$, there exist $y_{n} \in A_{n}(n=1,2, \ldots)$ such that $y_{n} \rightarrow y_{0}$. By (3.3), it follows from $y_{n} \in A_{n}$ that

$$
\phi_{n}\left(x_{n}, y_{n}\right) \notin-\operatorname{int} C \text {. }
$$

Since $\phi$ is $C$-upper semi-continuous on $X \times X$ and $x_{n} \rightarrow x^{*}, y_{n} \rightarrow y_{0}$, there exists $N_{1} \geq$ $N\left(\epsilon_{0}\right)$ such that

$$
\phi\left(x_{n}, y_{n}\right)-\phi\left(x^{*}, y_{0}\right) \in B^{\circ}\left(\epsilon_{0}\right)-C, \quad \forall n \geq N_{1}
$$

From (3.1), we derive

$$
\phi_{n}\left(x_{n}, y_{n}\right)-\phi\left(x_{n}, y_{n}\right) \in B\left(\epsilon_{0}\right), \quad \forall n \geq N_{1}
$$

By (3.4), (3.6), and (3.7), we obtain for all $n \geq N_{1}$,

$$
\begin{aligned}
\phi_{n}\left(x_{n}, y_{n}\right) & =\phi\left(x^{*}, y_{0}\right)+\left[\phi_{n}\left(x_{n}, y_{n}\right)-\phi\left(x_{n}, y_{n}\right)\right]+\left[\phi\left(x_{n}, y_{n}\right)-\phi\left(x^{*}, y_{0}\right)\right] \\
& \in \phi\left(x^{*}, y_{0}\right)+B\left(\epsilon_{0}\right)+B^{\circ}\left(\epsilon_{0}\right)-C \\
& \subset \phi\left(x^{*}, y_{0}\right)+B^{\circ}\left(2 \epsilon_{0}\right)-C \\
& \subset-\operatorname{int} C-C \subset-\operatorname{int} C,
\end{aligned}
$$

which contradicts (3.5). Hence $\phi\left(x^{*}, y\right) \notin-\operatorname{int} C$ for all $y \in A$. Thus we have shown $u:=$ $(\phi, A) \in M$. Consequently, the inequality (3.1) and $h\left(A_{n}, A\right) \rightarrow 0$ imply that $\lim _{n \rightarrow \infty} \rho\left(u_{n}\right.$, $u)=0$. Therefore, $(M, \rho)$ is a complete metric space.

Lemma 3.2 Let $f: X \rightarrow H$ be $C$-upper semi-continuous on $X$, then the set $L:=\{x \in X$ : $f(x) \notin-\operatorname{int} C\}$ is closed in $X$.

Proof Let $x_{n} \in L$ with $x_{n} \rightarrow x \in X$. We only need to prove $x \in L$. Assume, by contradiction, that $x \notin L$, then $f(x) \in-\operatorname{int} C$. Note that $-\operatorname{int} C$ is open, there exists $\epsilon>0$ such that $f(x)+$ $B^{\circ}(\epsilon) \subset-\operatorname{int} C$. Since $f: X \rightarrow H$ is $C$-upper semi-continuous at $x$ and $x_{n} \rightarrow x$, there exists $N>0$ such that, for any $n>N$, we have $f\left(x_{n}\right) \in f(x)+B^{\circ}(\epsilon)-C \subset-\operatorname{int} C-C \subset-\operatorname{int} C$. But it follows from $x_{n} \in L$ that $f\left(x_{n}\right) \notin-\operatorname{int} C$, which is a contradiction. The proof is complete.

For each $u=(\phi, A) \in M$, by the definition of $M, \operatorname{VEP}(\phi)$ must have at least one solution in $A$, i.e., $\exists x^{*} \in A$ such that $\phi\left(x^{*}, y\right) \notin-\operatorname{int} C$ for all $y \in A$. Denote by $S(u)$ the set of all solutions to $\operatorname{VEP}(\phi)$ in $A$. Then the correspondence $u \rightarrow S(u)$ yields a set-valued mapping $S: M \rightarrow 2^{X}$.

Lemma 3.3 $S: M \rightarrow 2^{X}$ is a usco mapping.

Proof For each $u=(\phi, A) \in M$, note that

$$
S(u)=\{x \in A: \phi(x, y) \notin-\operatorname{int} C, \forall y \in A\}=\bigcap_{y \in A}\{x \in A: \phi(x, y) \notin-\operatorname{int} C\} .
$$


Since $\phi$ is $C$-upper semi-continuous on $X \times X$, it is also $C$-upper semi-continuous on $A \times A$. Moreover, $x \rightarrow \phi(x, y)$ is also $C$-upper semi-continuous on $A$. By Lemma 3.2, for each $y \in A$, the set $\{x \in A: \phi(x, y) \notin-\operatorname{int} C\}$ is closed in $A$. Thus $S(u)$ is closed in $A$. Furthermore, $S(u)$ is compact since $A$ is compact.

Next, we will prove that $S$ is upper semi-continuous on $M$. We assume, by contradiction, that there exists $u=(\phi, A) \in M$ such that $S$ is not upper semi-continuous at $u$, then there exists an open neighborhood $G$ in $X$ with $G \supset S(u)$ such that, for each $n=1,2, \ldots$ and each open neighborhood $U_{n}=\left\{u^{\prime}=\left(\phi^{\prime}, A^{\prime}\right) \in M: \rho\left(u^{\prime}, u\right)<\frac{1}{n}\right\}$ of $u$, there exist $u_{n}=\left(\phi_{n}, A_{n}\right) \in$ $U_{n}$ and $x_{n} \in S\left(u_{n}\right)$ but $x_{n} \notin G$.

Since $u_{n}=\left(\phi_{n}, A_{n}\right) \in U_{n}$ for each $n=1,2, \ldots$, we have $\rho\left(u_{n}, u\right)<\frac{1}{n} \rightarrow 0$. Then

$$
\phi_{n} \rightarrow \phi \quad \text { and } \quad A_{n} \rightarrow A \text {. }
$$

It follows from $x_{n} \in S\left(u_{n}\right)$ that $x_{n} \in A_{n}$ and

$$
\phi_{n}\left(x_{n}, y\right) \notin-\operatorname{int} C, \quad \forall y \in A_{n} .
$$

By Lemma 2.7(i), $\bigcup_{n=1}^{+\infty} A_{n} \cup A$ is compact due to the compactness of $A_{n}$ and $A$. Note that $\left\{x_{n}\right\}_{n=1}^{+\infty} \subset \bigcup_{n=1}^{+\infty} A_{n} \cup A$. Without loss of generality, we suppose that $\left\{x_{n}\right\}_{n=1}^{+\infty}$ is convergent. Moreover, by Lemma 2.7(ii), the limit $x^{*}$ of $\left\{x_{n}\right\}_{n=1}^{+\infty}$ belongs to $A$, i.e., $x_{n} \rightarrow x^{*} \in A$. Meanwhile, $x_{n} \notin G$ and $G$ is open, thus $x^{*} \notin G$. Since $S(u) \subset G$, we have $x^{*} \notin S(u)$. Consequently, there exists $y \in A$ such that $\phi\left(x^{*}, y\right) \in-\operatorname{int} C$. Note that $-\operatorname{int} C$ is open; then there exists $\epsilon>0$ such that

$$
\phi\left(x^{*}, y\right)+B^{\circ}(\epsilon) \subset-\operatorname{int} C .
$$

Since $A_{n} \rightarrow A$ and $y \in A$, there exists a sequence $\left\{y_{n}\right\}_{n=1}^{+\infty}$ such that $y_{n} \in A_{n}$ and $y_{n} \rightarrow y$. Since $\phi_{n} \rightarrow \phi$, there exists $N_{1}>0$ such that, for any $n \geq N_{1}$,

$$
\phi_{n}\left(x_{n}, y_{n}\right)-\phi\left(x_{n}, y_{n}\right) \in B^{\circ}\left(\frac{\epsilon}{2}\right) .
$$

Moreover, $\phi$ is $C$-upper semi-continuous on $X \times X$ as well as $x_{n} \rightarrow x^{*}, y_{n} \rightarrow y$, hence there exists $N_{2}>N_{1}$ such that, for any $n \geq N_{2}$,

$$
\phi\left(x_{n}, y_{n}\right)-\phi\left(x^{*}, y\right) \in B^{\circ}\left(\frac{\epsilon}{2}\right)-C .
$$

By (3.10)-(3.12), we have for any $n \geq N_{2}$,

$$
\begin{aligned}
\phi_{n}\left(x_{n}, y_{n}\right) & =\phi\left(x^{*}, y\right)+\left[\phi_{n}\left(x_{n}, y_{n}\right)-\phi\left(x_{n}, y_{n}\right)\right]+\left[\phi\left(x_{n}, y_{n}\right)-\phi\left(x^{*}, y\right)\right] \\
& \in \phi\left(x^{*}, y\right)+B^{\circ}\left(\frac{\epsilon}{2}\right)+B^{\circ}\left(\frac{\epsilon}{2}\right)-C \\
& =\phi\left(x^{*}, y\right)+B^{\circ}(\epsilon)-C \subset-\operatorname{int} C-C \subset-\operatorname{int} C,
\end{aligned}
$$

which is in contradiction with (3.9). Therefore, $S$ must be upper semi-continuous on $M$. The proof is thus complete. 
Theorem 3.4 There exists a dense everywhere residual subset $Q$ of $M$ such that $S(u)$ is a singleton for each $u=(\phi, A) \in Q$, that is, $\operatorname{VEP}(\phi)$ has a unique solution in $A$.

Proof By Lemma 3.1, $M$ is a complete metric space, so it is a Baire space. Since $S: M \rightarrow 2^{X}$ is a usco mapping (Lemma 3.3) and $X$ is a metric subspace, by Lemma 2.5 , there exists a dense everywhere residual subset $Q$ of $M$ such that $S$ is lower semi-continuous at each $u=(\phi, A) \in Q$.

Assume, by contradiction, that $S\left(u_{0}\right)$ is not a singleton for some $u_{0}=\left(\phi_{0}, A_{0}\right) \in Q$. Then there exist at least two points $x_{1}, x_{2} \in S\left(u_{0}\right) \subset A_{0}$ with $x_{1} \neq x_{2}$. Consequently, there exist two open subsets $U$ and $V$ in $X$ such that $x_{1} \in U, x_{2} \in V$ and $U \cap V=\emptyset$.

Define a function $g: X \rightarrow \mathbb{R}$ as follows:

$$
g(x)=\frac{d\left(x, x_{1}\right)}{d\left(x, x_{1}\right)+d(x, X \backslash U)}, \quad \forall x \in X,
$$

where $d$ is the metric on $X$. Note that $g$ is continuous on $X$; $0 \leq g(x) \leq 1$ for all $x \in X$; $g(x)=0$ if and only if $x=x_{1} ; g(x)=1$ for all $x \in V$.

Take $z \in-\operatorname{int} C$. For each $n=1,2, \ldots$, let $\phi_{n}: X \times X \rightarrow H$ be defined by

$$
\phi_{n}(x, y)=\phi_{0}(x, y)+\left[\frac{1}{n} g(x)\right] z, \quad \forall x, y \in X .
$$

Furthermore, define

$$
u_{n}=\left(\phi_{n}, A_{0}\right) .
$$

For each $n=1,2, \ldots$, we will prove $u_{n} \in M$.

(i) It is easy to check that $\phi_{n}$ is $C$-upper semi-continuous on $X \times X$;

(ii) For any $x, y \in X$ with $x \neq y$, suppose $\phi_{n}(x, y) \notin-\operatorname{int} C$. Then we can claim that $\phi_{0}(x, y) \notin-\operatorname{int} C$. Otherwise $\phi_{0}(x, y) \in-\operatorname{int} C$. Note that $\left[\frac{1}{n} g(x)\right] z \in-C$, then

$$
\phi_{n}(x, y)=\phi_{0}(x, y)+\left[\frac{1}{n} g(x)\right] z \in-\operatorname{int} C-C \subset-\operatorname{int} C,
$$

which is a contradiction. By the $C$-strictly-quasi-monotonicity of $\phi_{0}$ and $\phi_{0}(x, y) \notin-\operatorname{int} C$, we get $\phi_{0}(y, x) \in-C$. Hence

$$
\phi_{n}(y, x)=\phi_{0}(y, x)+\left[\frac{1}{n} g(y)\right] z \in-C-C \subset-C .
$$

That is, $\phi_{n}$ is $C$-strictly-quasi-monotone on $X \times X$.

(iii) $\sup _{(x, y) \in X \times X}\left\|\phi_{n}(x, y)\right\| \leq \sup _{(x, y) \in X \times X}\left\|\phi_{0}(x, y)\right\|+\frac{1}{n} \cdot\|z\|<+\infty$.

(iv) From $x_{1} \in S\left(u_{0}\right)$ and $g\left(x_{1}\right)=0$, we derive

$$
\phi_{n}\left(x_{1}, y\right)=\phi_{0}\left(x_{1}, y\right)+\left[\frac{1}{n} g\left(x_{1}\right)\right] z=\phi_{0}\left(x_{1}, y\right) \notin-\operatorname{int} C, \quad \forall y \in A_{0},
$$

which implies $x_{1} \in S\left(u_{n}\right) \neq \emptyset$. 
Thus we have shown $u_{n} \in M$ for each $n=1,2, \ldots$. Consequently, $\rho\left(u_{n}, u_{0}\right) \leq \frac{1}{n} \cdot\|z\| \rightarrow 0$ as $n \rightarrow \infty$.

Note that $x_{2} \in V \cap S\left(u_{0}\right)$, then $V \cap S\left(u_{0}\right) \neq \emptyset$. Since $S$ is lower semi-continuous at $u_{0}$ and $u_{n} \rightarrow u_{0}$, there exists a positive integer $n_{0}>0$ big enough such that $V \cap S\left(u_{n_{0}}\right) \neq \emptyset$. Take $x_{n_{0}} \in V \cap S\left(u_{n_{0}}\right)$, then we have $x_{n_{0}} \in V \cap A_{0}, g\left(x_{n_{0}}\right)=1$ and $\phi_{n_{0}}\left(x_{n_{0}}, y\right) \notin-$ int $C$ for any $y \in A_{0}$. Take $y=x_{1}\left(\in A_{0}\right)$, then we get

$$
\phi_{0}\left(x_{n_{0}}, x_{1}\right)+\frac{1}{n_{0}} z=\phi_{0}\left(x_{n_{0}}, x_{1}\right)+\left[\frac{1}{n_{0}} g\left(x_{n_{0}}\right)\right] z=\phi_{n_{0}}\left(x_{n_{0}}, x_{1}\right) \notin-\operatorname{int} C .
$$

Note that $\frac{1}{n_{0}} z \in-\operatorname{int} C$. If $\phi_{0}\left(x_{n_{0}}, x_{1}\right) \in-C$, then $\phi_{n_{0}}\left(x_{n_{0}}, x_{1}\right) \in-C-\operatorname{int} C \subset-\operatorname{int} C$, which contradicts (3.13). Hence we have

$$
\phi_{0}\left(x_{n_{0}}, x_{1}\right) \notin-C
$$

Since $x_{1} \in S\left(u_{0}\right)$, we have $\phi_{0}\left(x_{1}, y\right) \notin-\operatorname{int} C$ for any $y \in A_{0}$. Taking $y=x_{n_{0}}\left(\in A_{0}\right)$, we get $\phi_{0}\left(x_{1}, x_{n_{0}}\right) \notin-\operatorname{int} C$. It follows from the $C$-strictly-quasi-monotonicity of $\phi_{0}$ that $\phi_{0}\left(x_{n_{0}}, x_{1}\right) \in-C$, which is in contradiction with (3.14). Therefore, $S(u)$ must be a singleton for each $u \in Q$.

When $H=\mathbb{R}, C=[0,+\infty)$, we get Corollary 3.5 as follows.

Corollary 3.5 Let

$$
M^{\prime}=\left\{\begin{array}{c}
f: X \times X \rightarrow \mathbb{R} \text { is upper semi-continuous on } X \times X ; \\
f \text { is pseudo-monotone on } X \times X ; \\
u=(f, A): \sup _{(x, y) \in X \times X}|f(x, y)|<+\infty ; \\
\text { A is a nonempty compact subset of } X ; \\
\text { and } \exists x \in A \text { such that } f(x, y) \geq 0, \forall y \in A
\end{array}\right\},
$$

where $f$ is called pseudo-monotone (see [30]) on $X \times X$ iff for any $x, y \in X$ with $x \neq y$,

$$
f(x, y) \geq 0 \Rightarrow f(y, x) \leq 0 .
$$

Then there exists a dense everywhere residual subset $Q^{\prime}$ of $M^{\prime}$ such that, for each $u=(f, A) \in$ $Q^{\prime}, f$ has a unique equilibrium point in $A$.

Remark 3.6 Corollary 3.5 generalized Theorem 3.2 of [9], one of main results of [9], as regards the following four aspects:

(i) we do not require the convexity of function $f \in M^{\prime}$;

(ii) we do not require the convexity and linear structure of the set $X$;

(iii) we omit the requirement that $f(x, x) \geq 0$ for all $x \in X$;

(iv) we replace the monotonicity of $f$ by pseudo-monotonicity which is weaker than the former.

\section{Well-posedness of VEPs}

As is well known, the notions of well-posedness can be mainly divided into three groups, namely, Hadamard type, Tykhonov type and Levitin-Polyak type. Generally speaking, to 
consider Tykhonov well-posedness of a problem, one introduces the notion of 'approximating sequence' for the solution and requires some convergence of such sequences to a solution of the problem; while, Hadamard well-posedness of a problem means the continuous dependence of the solutions on the data or the parameter of the problem; as for Levitin-Polyak well-posedness, we mean the convergence of the approximating solution sequence to a solution of the problem with some constraints; for more details to see [1115]. In this section, we will investigate the Hadamard well-posedness of VEPs.

Definition 4.1 Let $u \in M$. (1) The VEP associated with $u$ is said to be generalized Hadamard well-posed iff for any $u_{n} \in M$ and any $x_{n} \in S\left(u_{n}\right), u_{n} \rightarrow u$ implies that $\left\{x_{n}\right\}$ has a subsequence converging to an element of $S(u)$; (2) The VEP associated with $u$ is said to be Hadamard well-posed iff $S(u)=\{x\}$ (a singleton) and for any $u_{n} \in M$ and any $x_{n} \in S\left(u_{n}\right)$, $u_{n} \rightarrow u$ implies that $\left\{x_{n}\right\}$ converges to $x$.

Theorem 4.2 For each $u=(\phi, A) \in M$, the VEP associated with $u$ is generalized Hadamard well-posed.

Proof Let $u_{n}=\left(\phi_{n}, A_{n}\right) \in M, x_{n} \in S\left(u_{n}\right)$, and $u_{n} \rightarrow u$. Note that $A_{n} \rightarrow A$ because $u_{n} \rightarrow u$. According to Lemma 2.7(i), it follows from the compactness of $A_{n}$ and $A$ that $\bigcup_{n=1}^{+\infty} A_{n} \cup A$ is compact. Since $x_{n} \in S\left(u_{n}\right) \subset \bigcup_{n=1}^{+\infty} A_{n} \cup A$, there exists a convergent subsequence $\left\{x_{n_{k}}\right\}$ of $\left\{x_{n}\right\}$. Moreover, by Lemma 2.7(ii), the limit point $x^{*}$ of $\left\{x_{n_{k}}\right\}$ belongs to $A$, i.e., $x_{n_{k}} \rightarrow x^{*} \in A$. By Lemma 3.3, $S$ is upper semi-continuous at $u$ and $S(u)$ is compact.

If $x^{*} \notin S(u)$, then there exists an open set $O$ in $X$ such that $O \supset S(u)$ and $x^{*} \notin \bar{O}$. Since $S$ is upper semi-continuous at $u$ and $u_{n} \rightarrow u$, there is a positive integer $N$ such that $O \supset S\left(u_{n}\right)$ for all $n \geq N$. From $x_{n_{k}} \in S\left(u_{n_{k}}\right) \subset O$ for all $n_{k} \geq N$ and $x_{n_{k}} \rightarrow x^{*}$, it follows that $x^{*} \in \bar{O}$, which is a contradiction. Hence $x^{*} \in S(u)$. The proof is complete.

Theorem 4.3 There exists a dense everywhere residual subset $Q$ of $M$ such that, for each $u=(\phi, A) \in Q$, the VEP associated with $u$ is Hadamard well-posed, that is, VEPs in M are generic Hadamard well-posed.

Proof By Theorem 3.4, there exists a dense everywhere residual subset $Q$ of $M$ such that, for each $u \in Q, S(u)$ is a singleton.

Let $u=(\phi, A) \in Q$ and $S(u)=\{x\}$. Suppose $u_{n} \in M, x_{n} \in S\left(u_{n}\right)$, and $u_{n} \rightarrow u$. We shall prove $x_{n} \rightarrow x$. If it is not true, then there exist an open neighborhood $O$ of $x$ and a subsequence $\left\{x_{n_{k}}\right\}$ such that $x_{n_{k}} \notin O$. By Theorem 4.2, the VEP associated $u$ is generalized Hadamard well-posed. Since $S(u)=\{x\},\left\{x_{n_{k}}\right\}$ has a subsequence converging to $x$, which contradicts $x_{n_{k}} \notin O$.

\section{A representation theorem of the solution set to VEPs}

In this section, we use the limits of the solutions to VEPs, each of which has a unique solution, to provide an interesting representation of the solution set of each VEP in $M$, which in forms is very similar with the Clarke subdifferentials of the local Lipschitz functions.

Denote by

$$
P:=\{u=(\phi, A) \in M: S(u) \text { is a singleton }\} .
$$


By Theorem 3.4, $P \neq \emptyset$. It is clear that $P$ is the largest dense everywhere residual subset (ordered by the set inclusion) of $M$ such that, for each $u \in P, S(u)$ is a singleton and the VEP associated with $u$ is Hadamard well-posed.

Theorem 5.1 For each $u=(\phi, A) \in M$,

$$
S(u)=\left\{\lim _{n} x_{n}: x_{n} \in S\left(u_{n}\right), u_{n} \in P, u_{n} \rightarrow u\right\} .
$$

Proof The right-hand side of (5.1) means that we only consider such sequences $\left\{x_{n}\right\}$ and $\left\{u_{n}\right\}$ satisfying that $\left\{u_{n}\right\} \subset P ;\left\{u_{n}\right\}$ converges to $u ; x_{n}$ is the unique point of $S\left(u_{n}\right)$; and that $\left\{x_{n}\right\}$ is convergent.

Since $P$ is dense in $M$ and $u=(\phi, A) \in M$, there exists $\left\{u_{n}=\left(\phi_{n}, A_{n}\right)\right\} \subset P$ such that $\left\{u_{n}\right\}$ converges to $u$. By the definition of $P, S\left(u_{n}\right)$ has a unique point, denoted by $x_{n}$. Note that $\left\{x_{n}\right\} \subset \bigcup_{n=1}^{+\infty} A_{n} \cup A$, as well as $A_{n}$ and $A$ are compact and $A_{n} \rightarrow A$, due to Lemma 2.7, $\left\{x_{n}\right\}$ or its subsequence converges to a point of $A$. Hence the right-hand side set of (5.1) is well-defined and nonempty.

First, suppose $u_{n} \in P, x_{n} \in S\left(u_{n}\right), u_{n} \rightarrow u$ and $x_{n} \rightarrow x$. By Theorem 4.2, the VEP associated with $u$ is generalized Hadamard well-posed. It follows from $x_{n} \rightarrow x$ that $x \in S(u)$. Hence $S(u) \supset\left\{\lim _{n} x_{n}: x_{n} \in S\left(u_{n}\right), u_{n} \in P, u_{n} \rightarrow u\right\}$.

Next, let $x^{*} \in S(u)$. Define a function $g: X \rightarrow \mathbb{R}$ as follows:

$$
g(x)=\frac{d\left(x, x^{*}\right)}{1+d\left(x, x^{*}\right)}, \quad \forall x \in X
$$

where $d$ is the metric on $X$. Note that $g$ is continuous on $X ; 0 \leq g(x)<1$ for all $x \in X$; $g(x)=0$ if and only if $x=x^{*}$.

Take $z \in-\operatorname{int} C$. For each $n=1,2, \ldots$, define

$$
\phi_{n}(x, y):=\phi(x, y)+\left[\frac{1}{n} g(x)\right] z, \quad \forall x, y \in X \quad \text { and } \quad u_{n}:=\left(\phi_{n}, A\right) .
$$

Same as in the proof of Theorem 3.4, one can check that $u_{n} \in M$ and $x^{*} \in S\left(u_{n}\right)$ for each $n=1,2, \ldots$, and that $u_{n} \rightarrow u$ as $n \rightarrow \infty$. Moreover, we shall show that $S\left(u_{n}\right)$ is a singleton, and hence $S\left(u_{n}\right)=\left\{x^{*}\right\}$ for each $n=1,2, \ldots$. By way of contradiction, assume that $S\left(u_{n_{0}}\right)$ is not a singleton for some $u_{n_{0}}=\left(\phi_{n_{0}}, A\right)$. Then there exists $x^{\prime} \in S\left(u_{n_{0}}\right) \subset A$ with $x^{\prime} \neq x^{*}$. It follows from $x^{*} \in S(u)$ that $\phi\left(x^{*}, x^{\prime}\right) \notin-\operatorname{int} C$. By the $C$-strictly-quasi-monotonicity of $\phi$, we get $\phi\left(x^{\prime}, x^{*}\right) \in-C$. Note that $g\left(x^{\prime}\right)>0$ and $\left[\frac{1}{n_{0}} g\left(x^{\prime}\right)\right] z \in-\operatorname{int} C$. Then

$$
\phi_{n_{0}}\left(x^{\prime}, x^{*}\right):=\phi\left(x^{\prime}, x^{*}\right)+\left[\frac{1}{n_{0}} g\left(x^{\prime}\right)\right] z \in-C-\operatorname{int} C \subset-\operatorname{int} C .
$$

But it follows from $x^{\prime} \in S\left(u_{n_{0}}\right)$ that $\phi_{n_{0}}\left(x^{\prime}, x^{*}\right) \notin-\operatorname{int} C$, which is a contradiction. Thus $S\left(u_{n}\right)=\left\{x^{*}\right\}$ for each $n=1,2, \ldots$.

Take $x_{n}=x^{*}$ for each $n=1,2, \ldots$, then we have $u_{n} \in P, u_{n} \rightarrow u, x_{n} \in S\left(u_{n}\right)$, and $x^{*}=$ $\lim _{n} x_{n}$. From the arbitrariness of $x^{*} \in S(u)$, we derive

$$
S(u) \subset\left\{\lim _{n} x_{n}: x_{n} \in S\left(u_{n}\right), u_{n} \in P, u_{n} \rightarrow u\right\} .
$$

Combining the above two parts, we get the conclusion. 


\section{Conclusions}

In this paper, we considered a class of vector equilibrium problems. By considering the perturbations of vector-valued functions and feasible sets, we proved that each of the problems is generalized Hadamard well-posed, and that in the sense of Baire category, most of the problems have unique solution and are Hadamard well-posed. As an application, an interesting representation theorem for the solution set to each of the problems was provided.

\section{Competing interests}

The authors declare that they have no competing interests.

\section{Authors' contributions}

All authors contributed equally to the writing of this paper. All authors read and approved the final manuscript.

\section{Author details}

'College of Science, Guizhou University, Guiyang, Guizhou 550025, China. ${ }^{2}$ School of Science, Beijing Jiaotong University, Beijing, 100044, China.

\section{Acknowledgements}

The first author was supported by the NSFC (11271098), the Science and Technology Foundation of Guizhou Province (20102133), and the Scientific Research Projects for the Introduced Talents of Guizhou University (201343). The third author was supported by the National Basic Research Program of China (2010CB732501) and the NSFC (71271021). The authors, therefore, acknowledge these supports.

Received: 1 December 2013 Accepted: 22 April 2014 Published: 09 May 2014

\section{References}

1. Giannessi, F (ed.): Vector Variational Inequalities and Vector Equilibria: Mathematical Theories. Kluwer Academic, Dordrecht (2000)

2. Chen, GY, Huang, XX, Yang, XQ: Vector Optimization: Set-Valued and Variational Analysis. Springer, Berlin (2005)

3. Khanh, PQ, Tung, LT: Local uniqueness of solutions to Ky Fan vector inequalities using approximations as derivatives. J. Optim. Theory Appl. 155, 840-854 (2012)

4. Kenderov, PS: Most of the optimization problems have unique solution. C. R. Acad. Bulgare Sci. 37, $297-299$ (1984)

5. Beer, G: On a generic optimization theorem of Petar Kenderov. Nonlinear Anal. TMA 12, $627-655$ (1988)

6. Kenderov, PS, Ribarska, NK: Most of the two person zero-sum games have unique solution. In: Workshop/Mini-Conference on Functional Analysis and Optimization, Canberra, pp. 73-82 (1988)

7. Tan, KK, Yu, J, Yuan, XZ: The uniqueness of saddle points. Bull. Pol. Acad. Sci., Math. 43, 119-129 (1995)

8. Milchtaich, I: Generic uniqueness of equilibrium in large crowding games. Math. Oper. Res. 25, 349-364 (2000)

9. Yu, J, Peng, DT, Xiang, SW: Generic uniqueness of equilibrium points. Nonlinear Anal. TMA 74, 6326-6332 (2011)

10. Peng, DT, Yu, J, Xiu, NH: Generic uniqueness theorems with some applications. J. Glob. Optim. 56, $713-725$ (2013)

11. Lucchetti, R, Revalski, JP (eds.): Recent Developments in Well-Posed Variational Problems. Kluwer Academic, Dordrecht (1995)

12. Yang, H, Yu, J: Unified approaches to well-posedness with some applications. J. Glob. Optim. 31, 371-381 (2005)

13. Lucchetti, R: Convexity and Well-Posed Problems. Springer, New York (2006)

14. Huang, XX, Yang, XQ: Generalized Levitin-Polyak well-posedness in constrained optimization. SIAM J. Optim. 17, 243-258 (2006)

15. Yu, J: On well-posed problems. Acta Math. Appl. Sin. 34, 1007-1022 (2011)

16. Fang, YP, Hu, R, Huang, NJ: Well-posedness for equilibrium problems and for optimization problems with equilibrium constraints. Comput. Math. Appl. 55, 89-100 (2008)

17. Kimura, K, Liou, Y-C, Wu, S-Y, Yao, J-C: Well-posedness for parametric vector equilibrium with applications. J. Ind. Manag. Optim. 4, 313-327 (2008)

18. Bianchi, M, Kassay, G, Pini, R: Well-posedness for vector equilibrium problems. Math. Methods Oper. Res. 70, 171-182 (2009)

19. Li, SJ, Li, MH: Levitin-Polyak well-posedness of vector equilibrium problems. Math. Methods Oper. Res. 69, 125-140 (2009)

20. Salamon, J: Closedness and Hadamard well-posedness of the solution map for parametric vector equilibrium problems. J. Glob. Optim. 47, 173-183 (2010)

21. Peng, JW, Wang, Y, Wu, SY: Levitin-Polyak well-posedness of generalized vector equilibrium problems. Taiwan. J. Math. 15, 2311-2330 (2011)

22. Yang, $\mathrm{H}, \mathrm{Yu}$, J: Essential components of the set of weakly Pareto-Nash equilibrium points. Appl. Math. Lett. 15, 553-560 (2002)

23. Fan, K: A minimax inequality and applications. In: Shisha, O (ed.) Inequalities III, pp. 103-113. Academic Press, New York (1972)

24. Tan, KK, Yu, J, Yuan, XZ: The stability of Ky Fan's points. Proc. Am. Math. Soc. 123, 1511-1519 (1995)

25. Blum, E, Oettli, W: From optimization and variational inequalities to equilibrium problems. Math. Stud. 63, 123-135 (1994)

26. Aliprantis, CD, Border, KC: Infinite Dimensional Analysis, 3rd edn. Springer, Berlin (2006) 
27. Fort, MK: Points of continuity of semi-continuous functions. Publ. Math. (Debr.) 2, 100-102 (1951)

28. Yu, J: Essential weak efficient solution in multiobjective optimization problems. J. Math. Anal. Appl. 166, 230-235 (1992)

29. Xiang, SW, Yin, WS: Stability results for efficient solutions of vector optimization problems. J. Optim. Theory Appl. 134 385-398 (2007)

30. Bianchi, M, Schaible, S: Equilibrium problems under generalized convexity and generalized monotonicity. J. Glob. Optim. 30, 121-134 (2004)

10.1186/1687-1812-2014-115

Cite this article as: Peng et al.: The uniqueness and well-posedness of vector equilibrium problems with a representation theorem for the solution set. Fixed Point Theory and Applications 2014, 2014:115

Submit your manuscript to a SpringerOpen ${ }^{\circ}$ journal and benefit from:

- Convenient online submission

- Rigorous peer review

- Immediate publication on acceptance

- Open access: articles freely available online

- High visibility within the field

- Retaining the copyright to your article 